\title{
Endothelial-stromal interactions in angiogenesis
}

\author{
Christopher C. W. Hughes \\ Department of Molecular Biology \& Biochemistry, University of California Irvine, Irvine, CA 92697
}

\begin{abstract}
Purpose of review-Angiogenesis occurs in the context of a wound or tumor stroma. This review will focus on recent findings on the interactions between angiogenic endothelial cells and the other components of the stroma - fibroblasts, pericytes and extracellular matrix.

Recent findings - Large-scale gene expression arrays have provided a remarkable insight into the diversity of fibroblasts in different tissues and under different conditions. These somewhat neglected cells are now understood to play a critical role in tumor growth, regulating not only the phenotype of the tumor cells but also the angiogenic response that supports them. These advances are leading to an understanding of the soil and seed hypothesis at the molecular level. In addition there is a new focus on the role of pericytes in regulating angiogenesis and their potential as targets for tumor therapy.

Summary-Initiation of new blood vessel formation requires MMP induction leading to degradation of basement membrane, sprouting of endothelial cells, and regulation of pericyte attachment. Fibroblasts and their activated counterpart, the myofibroblast, play a large role in synchronizing these events through the expression of numerous extracellular matrix molecules, growth factors and morphogens, including FGFs and TGF $\beta$.
\end{abstract}

\section{Keywords}

Angiogenesis; endothelial; myofibroblast; pericyte; extracellular matrix; tumor stroma

\section{Introduction}

A concept now gaining wide acceptance is that understanding the nature of the tumor microenvironment is as important in the long-term treatment of cancer as understanding the genetic underpinnings of the cancer itself. Although originally conceived over a century ago as the "seed and soil" hypothesis (attributed to Stephen Paget [1]), we are only now beginning to understand the complex interplay between tumor cells (the seed) and the stroma, the tissue in which it grows (the soil). In the context of a tumor, stroma is defined as the surrounding extracellular matrix (ECM) and the mesenchymal cells within it, including endothelial cells (EC), fibroblasts, vascular smooth muscle cells (VSMC) and pericytes. In 1986 Dvorak made the important observation that tumor stroma looked very much like a wound, in that they shared active angiogenesis, numerous proliferating fibroblasts secreting

Correspondence to: Christopher C.W. Hughes, PhD, Department of Molecular Biology \& Biochemistry, McGaugh Hall, University of California Irvine, Irvine, CA 92697, T - +1-949 824 8771, F - +1-949 824 8551, cchughes@ uci.edu. 
a complex ECM, and all on a background of fibrin deposition [2]. Recently, this observation was given a molecular basis through the global analysis of gene expression in tumors and serum-stimulated fibroblasts [3]. This study reported a strong correlation between the serum response of fibroblasts and gene expression in prostate and hepatocellular carcinoma, and a somewhat more variable correlation between the serum response and gene expression in breast, lung and gastric carcinomas. Many excellent reviews have been published on the role of stroma in promoting tumor growth [4-7], therefore this review will focus specifically on the role of stroma in supporting angiogenesis, and particularly on interactions between: EC, ECM and matrix metalloproteinases (MMPs); EC and fibroblasts; and EC and pericytes.

\section{Interactions between EC, matrix and MMPs}

Non-angiogenic EC are usually closely apposed to a complex basement membrane (BM) containing collagen IV, laminin, entactin/nidogen and perlecan - a heparan sulfate proteoglycan. Laminin exists in multiple isoforms and it is laminin $10(a 5 \beta 1 \gamma 1)$ that is particularly enriched in EC BM, and seems to correlate with quiescence as it is not present in developing, angiogenic vessels [8,9]. EC interact with ECM proteins largely through surface-expressed integrins: $\alpha_{1} \beta_{1}, \alpha_{2} \beta_{1}$ and $\alpha_{3} \beta_{1}$, all of which bind collagen and laminin; $\alpha_{6} \beta_{1}$, which binds laminin; $\alpha_{4} \beta_{1}$ and $\alpha_{5} \beta_{1}$, both of which bind fibronectin and fibrin; and $a_{v} \beta_{3}$ and $\alpha_{v} \beta_{5}$, which variously bind vitronectin, fibronectin, fibrin and laminin [10]. Collagen I and fibronectin are particularly enriched in the interstitium between vessels, and are usually engaged only during angiogenic sprouting. Similarly, fibrin is only present at wound sites and is strongly pro-angiogenic. Like wounds, tumors are also rich in fibrin. Engagement of integrins with ECM proteins induces two-way signaling: inside-out signaling, which is dependent upon talin, increases integrin affinity for ligand [11]; while outside-in signaling acts through focal adhesions and their associated adaptors and kinases to affect cytoskeletal rearrangements as well as gene expression [12]. Interestingly, in vitro, gels composed of either collagen I or fibrin are able to support angiogenic sprouting of EC, although different integrins are involved in each case $-\alpha_{v} \beta_{3}$ and $\alpha_{5} \beta_{1}$ in fibrin and $\alpha_{2} \beta_{1}$ in collagen $[13,14]$. Matrigel is a widely-used extracellular matrix preparation derived from the mouse Engelbreth-Holm-Swarm sarcoma, and is enriched in collagen IV, nidogen and laminin, although which isoform has not been reported. Although EC will align into cords ("tubules") within a few hours when plated onto Matrigel, true intercellular lumens are not formed and the whole process is independent of RNA and protein synthesis. In addition, a number of non-endothelial cells, including fibroblasts and U87-MG glioblastoma cells, also align into similar cords on Matrigel [15].

Degradation and remodeling of the ECM is a critical component of angiogenesis and MMP expression by each of the mesenchymal cell types in a tumor/wound is necessary. EC must degrade the BM to allow migration into the surrounding tissue and further proteolysis is required to make space for lumen formation [16]. In an in vitro assay using collagen gels it was shown that lumen formation proceeds through the generation of intracellular vesicles that then fuse to form a vacuole, or proto-lumen. In turn, vacuoles in adjacent cells fuse, generating intercellular lumens. Knockdown by siRNA of MT1-MMP, but not MT3-MMP blocks lumen formation in this model [17]. An identical process of lumen formation has also been demonstrated during development of the intersegmental vessels in zebrafish [18], 
although the role of particular MMPs has not yet been determined. MT1-MMP has been shown in vivo to localize to endothelial tip cells - the cells that lead the developing sprout [19-21**]. This restricted expression allows for precise matrix degradation at the advancing front, while matrix rebuilding can occur more distally, where lumen formation and new BM assembly is occurring. Interestingly, restricted expression of MT1-MMP required the presence of VSMC - in their absence expression was widespread throughout the vessel. Gene deletion and other studies have also emphasized the important role of MMP2 and MMP9 in angiogenesis, and in particular a role for MMP9 in the angiogenic switch [22]. The activity of MMP2 and MMP9 is also spatially restricted, in this case through their capture at the cell surface by $\beta_{3}$ integrin and CD44, respectively [23]. Given the important role of MMPs in angiogenesis and tumor cell mobilization it is surprising that the numerous MMP inhibitors tested in clinical trials fared so poorly. A likely explanation is that MMPs can also generate anti-angiogenic molecules, such as angiostatin and tumstatin, the loss of which could abrogate the positive effects of MMP inhibition [24].

While an important role for MMPs is in the degradation of ECM, a perhaps equally important role is in the release of bound growth factors, such as vascular endothelial growth factor (VEGF), from the matrix. For example, a fraction of $\mathrm{VEGF}_{165}$ in tissue is bound to heparan sulfate proteoglycans through its heparin-binding domain, however, active VEGF can be released through cleavage of the protein by MMP3 or MMP9 [25]. Release of VEGF allows for the growth of larger vessels, whereas a non-cleavable form results in thinner vessels with multiple branch points.

MMP activity is controlled by a family of secreted inhibitors, the Tissue Inhibitors of Metalloproteinases, TIMP1-4 [17]. TIMP2 has additional actions in that it is required for the activation of MMP2 by MT1-MMP, and can also signal to the EC through binding to the laminin receptor $\alpha_{3} \beta_{1}$ [26]. This binding inhibits the activity of growth factor receptor tyrosine kinases such as FGFR or VEGFR through the action of the phosphatase Shp-1. In addition, de novo synthesis of $\mathrm{p} 27^{\mathrm{Kip} 1}$ downregulates cdk 2 and cdk4, resulting in cell cycle arrest [27].

\section{Interactions between EC and fibroblasts}

Fibroblasts were original described over 100 years ago and are still largely defined by their location and what they are not - non-SMC, non-EC, non-epithelial cells of the stroma. Only fibroblast activation protein appears to be somewhat specific, and this lack of good markers has hampered in-depth investigations. A recent gene analysis study revealed that fibroblasts are quite different cells depending on the tissue from which they are isolated [28], although all have as a primary purpose the synthesis and maintenance of ECM. Interestingly, however, myofibroblasts from multiple different breast carcinomas had similar gene expression profiles [29]. In response to wounding, and in the setting of tumor growth, fibroblasts become activated and highly synthetic, secreting for example, collagen I and IV, fibronectin, heparan sulfate proteoglycans, secreted protein acidic and rich in cysteine (SPARC), tenascin and connective tissue growth factor (CTGF) [3,30]. These cells are recognized by their expression of a-smooth-muscle actin and are termed myofibroblasts in wound tissue, and have been called carcinoma-associated fibroblasts (CAFs) in tumors [7], where they 
may comprise up to $80 \%$ of the stromal fibroblasts in breast carcinomas [5]. Although tumor cells often secrete VEGF, the major source in most tumors is likely to be the fibroblasts [31]. VEGF, also called vascular permeability factor (VPF) is not only a morphogen for EC it also induces vascular leak. The subsequent deposition of fibrin provides a provisional matrix for angiogenic EC, and also acts as a chemoattractant for circulating endothelial progenitor cells, inflammatory cells, and resident fibroblasts.

TSP-1 is generally thought to be a negative regulator of angiogenesis, however it is released by platelets at fibrin-rich wound sites, where angiogenesis is active, and a recent study suggests that it can stimulate VEGF expression by dermal fibroblasts. VEGF then drives EC tubulogenesis [32]. In addition, TSP-1 also promotes EC migration by downregulating EC expression of PAI-1, a potent inhibitor of fibrinolysis.

Activation of fibroblasts is an important precursor to rapid tumor growth as can be seen in tumor xenograft experiments. When fibroblasts derived from tumor stroma (CAFs) are mixed with tumor cells, for example MCF7 breast carcinoma cells, and then injected into nude mice they generate larger and faster growing tumors than do MCF7 cells mixed with fibroblasts from normal, non-cancer, tissue [33]. At least some of this effect is due to the expression of SDF-1 by activated fibroblasts, which recruits circulating endothelial precursor cells (EPC), thereby promoting tumor angiogenesis, however fibroblast expression of HGF, IL-8, SPARC, and TGF $\beta$ is also likely to contribute [30]. In studies on pancreatic tumor stromal cells it was found that a non tumor-promoting phenotype correlated with low expression of CTGF, and that CTGF could be induced by TGF $\beta$ [34]. In admixing experiments, 3T3 fibroblasts expressing CTGF promoted more rapid growth of pancreatic tumor cells and an increased microvessel density, when compared to injections of tumor cells and control 3T3 cells [34]. Fibroblast-expressed CTGF acts as a depot for VEGF that can be rapidly mobilized as a result of CTGF cleavage by tumor-derived MMP7 [35*], or by EC-derived MMP9 [25]. Fibroblasts also regulate EC activation and proliferation through expression of syndecans. Syndecan-1 is a heparan sulfate proteoglycan expressed on fibroblasts in more than $70 \%$ of human breast carcinomas. Its expression on fibroblasts in a tumor transplant model significantly increased microvessel density and vessel area [36], although the mechanism is not known.

Numerous in vitro studies have examined the role of mesenchymal cells/fibroblasts in EC tubulogenesis [37-39], and in all cases their presence has been shown to promote sprouting, and especially, formation of intercellular lumens. A recent study directly examined the necessity for matrix deposition by fibroblasts in the promotion of EC tubulogenesis using reconstructed connective tissue [37]. It was found that reducing fibroblast ECM synthesis by removing ascorbate from the medium reduced tube formation by 10 -fold. There was no reduction in fibroblast or EC number, and there was no decrease in FGF2 synthesis. Remarkably, when the connective tissue was conditioned by fibroblasts, and the fibroblasts were then killed by repeated freeze-thaw, there was still strongly diminished tubulogenesis in freshly-added EC, even in the presence of fibroblast-conditioned medium. The implication is that living fibroblasts need to be in close association with the EC for tubulogenesis to occur, although, it is not clear that the conditioned medium contained sufficient VEGF to promote tubulogenesis. 
Earlier studies in vitro established a necessary role for fibroblast-derived factors in tubulogenesis [38], and these could be substituted by PMA [40], suggesting a role for PKC signaling in lumen formation. Recently it has been reported that $\mathrm{EC}$ tube formation in fibrin gels is much more strictly dependent on the distance of the EC from the fibroblasts than the distance of the $\mathrm{EC}$ from the culture medium, suggesting that the fibroblast-derived factor(s) is poorly diffusible, either due to size or to matrix interactions [41]. Indeed, there is evidence that collagen I synthesis by fibroblasts is a critical component of tube formation in fibrin, although it is not sufficient in the absence of fibroblasts (unpublished observations). Bone marrow-derived mesenchymal stem cells can also support tubulogenesis, and this appears to be dependent on expression of MT1-MMP [42]. Recent work has shed light on how crosstalk between stromal cells regulates MMP induction. In response to VEGF and other angiogenic factors, fibroblasts, EC and tumor cells upregulate and release extracellular matrix metalloproteinase inducer (EMMPRIN), which not only induces MMP expression by mesenchymal cells but also drives differentiation of fibroblasts to myofibroblasts [43,44].

Finally, recent work has shown that EC can undergo an EC-to-mesenchymal transition to generate myofibroblast-like cells. In response to TGF $\beta$ EC downregulate CD31 and upregulate fibroblast-specific protein-1 [45**]. In vivo studies using Tie2-CRE-mediated lacZ tagging of EC confirmed the endothelial origin of these myofibroblast-like cells in tumors.

\section{Interactions between EC and pericytes}

Pericytes and vascular smooth muscle cells likely emerge from the same cell lineage and both ensheath blood vessels. While pericytes are embedded in the basement membrane and make intimate contacts with the EC, VSMC in contrast lie largely outside of the basement membrane and make only minimal direct contact with EC [46]. Similar to fibroblasts there are few reliable markers for pericytes, although they do express desmin, a-smooth muscle actin, regulator of $G$ protein signaling 5 (RGS-5) and PDGFR $\beta$ [47,48]. Pericytes are essential for the stabilization of neovessels and recent data suggest that this may involve regulation of MMP activity [16**]. In an in vitro three-dimensional model of EC-pericyte interaction, cross-talk between the cells led to sprout stabilization as a result of induction of TIMP3 in the pericytes and TIMP2 in the EC. Knockdown of TIMP expression led to vessel regression in a process dependent on MMP1, MMP10 and ADAM15. The major target of TIMP2 and TIMP3 in this assay was MT1-MMP [16**]. Interestingly, at the tip of developing sprouts pericytes are absent and MT1-MMP expression is high, consistent with the need for matrix degradation at this location [21**]. Blocking Tie2 activity leads to reduced recruitment of VSMC/pericytes to the developing vessels and a consequent spread of MT1-MMP expression to the cells comprising the vessel trunk [21**]. EC-pericyte interaction also leads to activation of latent TGF $\beta$, which subsequently signals to block both $\mathrm{EC}$ and pericyte proliferation.

Several factors have been implicated in the recruitment of pericytes to the vessel wall, including sphingosine-1-phosphate, angiopoietin-1 (Ang-1) and PDGFB [49]. Ang-1 is made by VSMC and pericytes and binds to the Tie-2 receptor on EC, leading to tight ECpericyte interaction and vessel stabilization. Tie-2 signaling induces the expression and 
release of HB-EGF by EC, which binds to EGF receptors and promotes migration of mesenchymal cells, including pericytes [50]. Ang-2 also binds to Tie2 and can act antagonistically to Ang-1. Systemic overexpression of Ang-2 had the unexpected result of rapidly regressing tumors as a result of pericyte loss and destabilization of the vessels within the tumor [51*]. As a relaxing of the pericyte-EC interaction is usually a prelude to further rounds of angiogenesis, this complete loss of vessel integrity, in the continued presence of VEGF, is hard to explain. Indeed, when Ang-2 was overexpressed in retina it led to an increase in the density of the vascular network [52], consistent with controlled angiogenesis. Loss of the PDGF pathway, by gene deletion of ligand or PDGFR $\beta$, results in vascular aneurysms and multiple small hemorrhages, consequent to a loss of pericyte coverage [53]. Conversely, expression of a constitutively active form of the receptor PDGFR $\beta$ in embryonic stem (ES) cells leads to an abundant pericyte vascular coating when the ES cells are differentiated into embryoid bodies and induced to sprout vessels [54]. Surprisingly, overexpression of PDGFB in colorectal and pancreatic cancer cells led to reduced tumor growth, which was ascribed to an increase in mural cell coverage and increased vascular stability. Indeed, in follow-up experiments VSMC were found to decrease EC proliferation by $50 \%\left[55^{*}\right]$.

MMP9 is expressed by pericytes and is important for migration of the cells to the vessel wall. In animals deficient for MMP9, vessels growing into transplanted tumors showed a paucity of pericyte coverage, which could be alleviated by transfer of wild-type (MMP9positive) bone marrow [44]. Defective mural cell recruitment to vessels is also seen in the human disease CADASIL, which has been mapped to mutations in the notch3 gene [46].

\section{Conclusion}

Although EC have been the primary focus for angiogenesis research it is now clear that they function within a complex network of interactions. EC receive activation and stabilization signals from the local matrix environment, which they interact with through integrins and MMPs, and from various mesenchymal cells that with the ECM comprise the stroma. Not addressed here are local variations, dependent on the tissue type, in the phenotype of the fibroblasts, pericytes, and EC. An important area for future study will be determining the role of pericytes in stabilizing tumor vasculature. In some settings increasing the number of pericytes may stabilize and normalize blood vessels, allowing more effective delivery of tumor cell cytotoxics. Conversely, in other settings limiting pericyte coverage may lead to destabilization and collapse of the vasculature, thereby promoting tumor regression. Only when we understand all of the tissue-specific cross-talk between these components will we be able to rationally and successfully target angiogenesis in those tissues.

\section{Acknowledgements}

Supported by grants from the National Institutes of Health (RO1 HL60067 and RO1 HL086959). The author thanks Drs Matthew Holderfield and Martin Nakatsu for helpful discussions and critical reading of the manuscript. 


\section{References and recommended reading}

1. Paget S: The Distribution Of Secondary Growths In Cancer Of The Breast. The Lancet 1889, 133:571-573.

2. Dvorak HF: Tumors: wounds that do not heal. Similarities between tumor stroma generation and wound healing. N Engl J Med 1986, 315:1650-1659. [PubMed: 3537791]

3. Chang HY, Sneddon JB, Alizadeh AA, et al.: Gene expression signature of fibroblast serum response predicts human cancer progression: similarities between tumors and wounds. PLoS Biol 2004, 2:E7. [PubMed: 14737219]

4. Jodele S, Blavier L, Yoon JM, et al.: Modifying the soil to affect the seed: role of stromal-derived matrix metalloproteinases in cancer progression. Cancer Metastasis Rev 2006, 25:35-43. [PubMed: 16680570]

5. Kalluri R, Zeisberg M: Fibroblasts in cancer. Nat Rev Cancer 2006, 6:392-401. [PubMed: 16572188]

6. Laconi E: The evolving concept of tumor microenvironments. Bioessays 2007, 29:738-744. [PubMed: 17621638]

7. Orimo A, Weinberg RA: Stromal fibroblasts in cancer: a novel tumor-promoting cell type. Cell Cycle 2006, 5:1597-1601. [PubMed: 16880743]

8. Hallmann R, Horn N, Selg M, et al.: Expression and function of laminins in the embryonic and mature vasculature. Physiol Rev 2005, 85:979-1000. [PubMed: 15987800]

9. Sorokin LM, Pausch F, Frieser M, et al.: Developmental regulation of the laminin alpha5 chain suggests a role in epithelial and endothelial cell maturation. Dev Biol 1997, 189:285-300. [PubMed: 9299121]

10. Weis SM: Evaluating integrin function in models of angiogenesis and vascular permeability. Methods Enzymol 2007, 426:505-528. [PubMed: 17697897]

11. Ginsberg MH, Partridge A, Shattil SJ: Integrin regulation. Curr Opin Cell Biol 2005, 17:509-516. [PubMed: 16099636]

12. Del Pozo MA, Schwartz MA: Rac, membrane heterogeneity, caveolin and regulation of growth by integrins. Trends Cell Biol 2007, 17:246-250. [PubMed: 17363257]

13. Bayless KJ, Davis GE: Sphingosine-1-phosphate markedly induces matrix metalloproteinase and integrin-dependent human endothelial cell invasion and lumen formation in three-dimensional collagen and fibrin matrices. Biochem Biophys Res Commun 2003, 312:903-913. [PubMed: 14651957]

14. Carnevale E, Fogel E, Aplin AC, et al.: Regulation of postangiogenic neovessel survival by beta1 and beta3 integrins in collagen and fibrin matrices. J Vasc Res 2007, 44:40-50. [PubMed: 17167269]

15. Donovan D, Brown NJ, Bishop ET, et al.: Comparison of three in vitro human 'angiogenesis' assays with capillaries formed in vivo. Angiogenesis 2001, 4:113-121. [PubMed: 11806243]

**16. Saunders WB, Bohnsack BL, Faske JB, et al.: Coregulation of vascular tube stabilization by endothelial cell TIMP-2 and pericyte TIMP-3. J Cell Biol 2006, 175:179-191. [PubMed: 17030988] Using an elegant in vitro co-culture model the authors provide a convincing demonstration of the role of pericytes in regulating MMP activity around angiogenic EC. Crosstalk between pericytes and EC led to mutual induction of TIMPs and inhibition of MT1-MMP leading to vessel stabilization. These data emphasize the importance of local regulation of MMP activity.

17. Davis GE, Saunders WB: Molecular balance of capillary tube formation versus regression in wound repair: role of matrix metalloproteinases and their inhibitors. J Investig Dermatol Symp Proc 2006, 11:44-56.

18. Kamei M, Saunders WB, Bayless KJ, et al.: Endothelial tubes assemble from intracellular vacuoles in vivo. Nature 2006, 442:453-456. [PubMed: 16799567]

19. Gerhardt H, Golding M, Fruttiger M, et al.: VEGF guides angiogenic sprouting utilizing endothelial tip cell filopodia. J Cell Biol 2003, 161:1163-1177. [PubMed: 12810700] 
20. Sainson RC, Aoto J, Nakatsu MN, et al.: Cell-autonomous notch signaling regulates endothelial cell branching and proliferation during vascular tubulogenesis. Faseb J 2005, 19:1027-1029. [PubMed: 15774577]

**21. Yana I, Sagara H, Takaki S, et al.: Crosstalk between neovessels and mural cells directs the sitespecific expression of MT1-MMP to endothelial tip cells. J Cell Sci 2007, 120:1607-1614. [PubMed: 17405818] A combination of in vitro and in vivo studies are used to emphasize the importance of pericyte/VSMC coverage in affecting local EC phenotype. EC downregulate MT1MMP activity when closely apposed by mural cells, but where they are absent - at the migrating tip - MT1-MMP is active, allowing migration of the tip cell.

22. Bergers G, Brekken R, McMahon G, et al.: Matrix metalloproteinase-9 triggers the angiogenic switch during carcinogenesis. Nat Cell Biol 2000, 2:737-744. [PubMed: 11025665]

23. van Hinsbergh VW, Engelse MA, Quax PH: Pericellular proteases in angiogenesis and vasculogenesis. Arterioscler Thromb Vasc Biol 2006, 26:716-728. [PubMed: 16469948]

24. Hamano Y, Zeisberg M, Sugimoto H, et al.: Physiological levels of tumstatin, a fragment of collagen IV alpha3 chain, are generated by MMP-9 proteolysis and suppress angiogenesis via alphaV beta3 integrin. Cancer Cell 2003, 3:589-601. [PubMed: 12842087]

25. Lee S, Jilani SM, Nikolova GV, et al.: Processing of VEGF-A by matrix metalloproteinases regulates bioavailability and vascular patterning in tumors. J Cell Biol 2005, 169:681-691. [PubMed: 15911882]

26. Seo DW, Li H, Guedez L, et al.: TIMP-2 mediated inhibition of angiogenesis: an MMPindependent mechanism. Cell 2003, 114:171-180. [PubMed: 12887919]

27. Seo DW, Li H, Qu CK, et al.: Shp-1 mediates the antiproliferative activity of tissue inhibitor of metalloproteinase-2 in human microvascular endothelial cells. J Biol Chem 2006, 281:3711-3721. [PubMed: 16326706]

28. Chang HY, Chi JT, Dudoit S, et al.: Diversity, topographic differentiation, and positional memory in human fibroblasts. Proc Natl Acad Sci U S A 2002, 99:12877-12882. [PubMed: 12297622]

29. Allinen M, Beroukhim R, Cai L, et al.: Molecular characterization of the tumor microenvironment in breast cancer. Cancer Cell 2004, 6:17-32. [PubMed: 15261139]

30. Sato N, Maehara N, Goggins M: Gene expression profiling of tumor-stromal interactions between pancreatic cancer cells and stromal fibroblasts. Cancer Res 2004, 64:6950-6956. [PubMed: 15466186]

31. Fukumura D, Xavier R, Sugiura T, et al.: Tumor induction of VEGF promoter activity in stromal cells. Cell 1998, 94:715-725. [PubMed: 9753319]

32. Kellouche S, Mourah S, Bonnefoy A, et al.: Platelets, thrombospondin-1 and human dermal fibroblasts cooperate for stimulation of endothelial cell tubulogenesis through VEGF and PAI-1 regulation. Exp Cell Res 2007, 313:486-499. [PubMed: 17126831]

33. Orimo A, Gupta PB, Sgroi DC, et al.: Stromal fibroblasts present in invasive human breast carcinomas promote tumor growth and angiogenesis through elevated SDF-1/CXCL12 secretion. Cell 2005, 121:335-348. [PubMed: 15882617]

34. Yang F, Tuxhorn JA, Ressler SJ, et al.: Stromal expression of connective tissue growth factor promotes angiogenesis and prostate cancer tumorigenesis. Cancer Res 2005, 65:8887-8895. [PubMed: 16204060]

*35. Ito TK, Ishii G, Chiba H, et al.: The VEGF angiogenic switch of fibroblasts is regulated by MMP-7 from cancer cells. Oncogene 2007, 26:7194-7203. [PubMed: 17525740] Evidence that CTGF and MMP7 coordinate the release of VEGF as part of the angiogenic switch.

36. Maeda T, Desouky J, Friedl A: Syndecan-1 expression by stromal fibroblasts promotes breast carcinoma growth in vivo and stimulates tumor angiogenesis. Oncogene 2006, 25:1408-1412. [PubMed: 16247452]

37. Berthod F, Germain L, Tremblay N, et al.: Extracellular matrix deposition by fibroblasts is necessary to promote capillary-like tube formation in vitro. J Cell Physiol 2006, 207:491-498. [PubMed: 16453301]

38. Montesano R, Pepper MS, Orci L: Paracrine induction of angiogenesis in vitro by Swiss 3T3 fibroblasts. JCS 1993, 105:1013-1024. 
39. Nakatsu MN, Sainson RC, Aoto JN, et al.: Angiogenic sprouting and capillary lumen formation modeled by human umbilical vein endothelial cells (HUVEC) in fibrin gels: the role of fibroblasts and Angiopoietin-1 small star, filled. Microvasc Res 2003, 66:102-112. [PubMed: 12935768]

40. Montesano R, Orci L: Tumor-promoting phorbol ester induces angiogenesis in vitro. Cell 1985, 42:469-477. [PubMed: 2411423]

41. Griffith CK, Miller C, Sainson RC, et al.: Diffusion limits of an in vitro thick prevascularized tissue. Tissue Eng 2005, 11:257-266. [PubMed: 15738680]

42. Ghajar CM, Blevins KS, Hughes CC, et al.: Mesenchymal Stem Cells Enhance Angiogenesis in Mechanically Viable Prevascularized Tissues via Early Matrix Metalloproteinase Upregulation. Tissue Eng 2006.

43. Huet E, Vallee B, Szul D, et al.: Extracellular matrix metalloproteinase inducer/CD147 promotes myofibroblast differentiation by inducing $\{$ alpha $\}$-smooth muscle actin expression and collagen gel contraction: implications in tissue remodeling. Faseb J 2007.

44. Jodele S, Chantrain CF, Blavier L, et al.: The contribution of bone marrow-derived cells to the tumor vasculature in neuroblastoma is matrix metalloproteinase-9 dependent. Cancer Res 2005, 65:3200-3208. [PubMed: 15833851]

**45. Zeisberg EM, Potenta S, Xie L, et al.: Discovery of endothelial to mesenchymal transition as a source for carcinoma-associated fibroblasts. Cancer Res 2007, 67:10123-10128. [PubMed: 17974953] Convincing evidence is provided that endothelial cells can undergo an EC-tomesenchymal transition, thereby generating myofibroblast-like cells in tumors. The use of tie2CRE-mediated lacZ tagging convincingly demonstrates the EC origin of these cells. The authors suggest that anti-angiogenic therapies might also target this transition, thereby reducing the numbers of tumor-supporting fibroblasts.

46. Armulik A, Abramsson A, Betsholtz C: Endothelial/pericyte interactions. Circ Res 2005, 97:512523. [PubMed: 16166562]

47. Bergers G, Song S: The role of pericytes in blood-vessel formation and maintenance. Neuro Oncol 2005, 7:452-464. [PubMed: 16212810]

48. Bondjers C, Kalen M, Hellstrom M, et al.: Transcription profiling of platelet-derived growth factorB-deficient mouse embryos identifies RGS5 as a novel marker for pericytes and vascular smooth muscle cells. Am J Pathol 2003, 162:721-729. [PubMed: 12598306]

49. Chantrain CF, Henriet P, Jodele $S$, et al.: Mechanisms of pericyte recruitment in tumour angiogenesis: a new role for metalloproteinases. Eur J Cancer 2006, 42:310-318. [PubMed: 16406506]

50. Iivanainen E, Nelimarkka L, Elenius V, et al.: Angiopoietin-regulated recruitment of vascular smooth muscle cells by endothelial-derived heparin binding EGF-like growth factor. Faseb J 2003, 17:1609-1621. [PubMed: 12958167]

*51. Cao Y, Sonveaux P, Liu S, et al.: Systemic overexpression of angiopoietin-2 promotes tumor microvessel regression and inhibits angiogenesis and tumor growth. Cancer Res 2007, 67:38353844. [PubMed: 17440098] This study demonstrates that prolonged overexpression of Ang2 inhibits tumor growth by blocking pericyte recruitment to tumor vessels, thereby leading to their destabilization. Interestingly, inhibition of VEGF activity is not required.

52. Feng Y, vom Hagen F, Pfister F, et al.: Impaired pericyte recruitment and abnormal retinal angiogenesis as a result of angiopoietin-2 overexpression. Thromb Haemost 2007, 97:99-108. [PubMed: 17200776]

53. Lindahl P, Johansson BR, Leveen P, et al.: Pericyte loss and microaneurysm formation in PDGF-Bdeficient mice. Science 1997, 277:242-245. [PubMed: 9211853]

54. Magnusson PU, Looman C, Ahgren A, et al.: Platelet-derived growth factor receptor-beta constitutive activity promotes angiogenesis in vivo and in vitro. Arterioscler Thromb Vasc Biol 2007, 27:2142-2149. [PubMed: 17656670]

*55. McCarty MF, Somcio RJ, Stoeltzing O, et al.: Overexpression of PDGF-BB decreases colorectal and pancreatic cancer growth by increasing tumor pericyte content. J Clin Invest 2007, 117:2114-2122. [PubMed: 17641778] In contrast to the study of Cao et al. (Ref 51), this study shows that an increase in pericyte recruitment to tumor vasculature slows tumor growth. Blocking of the PDGF receptor led to a decrease in pericytes in the tumor and an increase in tumor growth. 
The implication of these two studies is that the balance of pericyte numbers is critical for optimal tumor growth - too many and the vessels are stabilized, thereby restricting sprouting, too few and the vessels are too unstable to support adequate blood flow. Either way reduces tumor growth. 